\title{
Existence of $T-v-p(x)$-Solution of a Nonhomogeneous Elliptic Problem with Right Hand Side Measure
}

\section{El Houcine Rami, Abdelkrim Barbara, El Houssine Azroul}

Laboratory LAMA, Department of Mathematics, Faculty of Sciences Dhar El Mahraz, Sidi Mohamed Ben Abdellah University, Fez, Morocco

Email: ramielhoucine@gmail.com, babdelkarim66@hotmail.com, elhoussine.azroul@gmail.com

How to cite this paper: Rami, El H., Barbara, A. and Azroul, El H. (2021) Existence of $T$ - $v-p(x)$-Solution of a Nonhomogeneous Elliptic Problem with Right Hand Side Measure. Journal of Applied Mathematics and Physics, 9, 2717-2732.

https://doi.org/10.4236/jamp.2021.911175

Received: September 18, 2021

Accepted: November 12, 2021

Published: November 15, 2021

Copyright $\odot 2021$ by author(s) and Scientific Research Publishing Inc. This work is licensed under the Creative Commons Attribution International License (CC BY 4.0).

http://creativecommons.org/licenses/by/4.0/

\begin{abstract}
Using the theory of weighted Sobolev spaces with variable exponent and the $L^{1}$-version on Minty's lemma, we investigate the existence of solutions for some nonhomogeneous Dirichlet problems generated by the Leray-Lions operator of divergence form, with right-hand side measure. Among the interest of this article is the given of a very important approach to ensure the existence of a weak solution of this type of problem and of generalization to a system with the minimum of conditions.
\end{abstract}

\section{Keywords}

Nonhomogeneous Elliptic Equations, Dirichlet Problems, Weighted Sobolev Spaces with Variable Exponent, Minty's Lemma, $T-v-p(x)$-Solutions

\section{Introduction}

Consider the nonhomogeneous and nonlinear Dirichlet boundary value problem:

$$
(\mathcal{P}) \begin{cases}-\operatorname{div}(a(x, u, \nabla u))=\mu & \text { in } \Omega \\ u=0 & \text { on } \partial \Omega,\end{cases}
$$

where $\Omega$ is a bounded open domain of $\mathbb{R}^{N} \quad(N \geq 2)$ and

$A u=-\operatorname{div}(a(x, u, \nabla u))$ is a Leray-Lions operator defined from the weighted Sobolev spaces with variable exponent $W_{0}^{1, p(x)}(\Omega, v)$ into its dual $W^{-1, p^{\prime}(x)}\left(\Omega, v^{*}\right)$ with $v^{*}=v^{1-p^{\prime}(x)}$ and $\frac{1}{p(x)}+\frac{1}{p^{\prime}(x)}=1$. The datum $\mu$ is a measure that admits an $L^{1}$-dual composition. 
Throughout the paper, we suppose that the exponent $p(\cdot)$ is an element of $C_{+}(\bar{\Omega})=\{\log$-Hölder continuous function $p(\cdot): \bar{\Omega} \rightarrow \mathbb{R}$ such that $\left.1<p_{-} \leq p(x) \leq p_{+}<N\right\}$ (where for all $h \in C_{+}(\bar{\Omega})$, we denote $h_{+}$and $h_{-}$by $h_{+}=\sup _{x \in \Omega} h(x)$ and $\left.h_{-}=\inf _{x \in \Omega} h(x)\right)$ and that $v$ is a weight function defined on $\Omega$ (i.e., $v$ is a measurable function which is strictly positive a.e. in $\Omega$ ) satisfying:

$$
\begin{gathered}
v \in L_{l o c}^{1}(\Omega), \\
v^{\frac{-1}{p(x)-1}} \in L_{l o c}^{1}(\Omega), \\
v^{-s(x)} \in L^{1}(\Omega) \text { for some } s(x) \in\left(\frac{N}{p(x)}, \infty\right) \cap\left(\frac{1}{p(x)-1}, \infty\right) .
\end{gathered}
$$

The problem $(\mathcal{P})$ is studied where the following assumptions are satisfied: $\left(H_{1}\right)$ a is a Carathéodory function satisfying:

$$
\begin{gathered}
|a(x, r, \xi)| \leq \beta v^{\frac{1}{p(x)}}\left[b(x)+|r|^{p(x)-1}+v^{\frac{1}{p^{\prime}(x)}}(\gamma(r)|\xi|)^{p(x)-1}\right] \\
{[a(x, r, \xi)-a(x, r, \eta)](\xi-\eta) \geq 0 \quad \forall \xi, \eta \in \mathbb{R}^{N}} \\
a(x, r, \xi) \xi \geq \alpha v|\xi|^{p(x)}
\end{gathered}
$$

where $b(\cdot)$ is a positive function in $L^{p^{\prime}(x)}(\Omega), \gamma(r)$ is a continuous function and $\alpha, \beta$ are strictly positive constants.

$\left(H_{2}\right)$ The second member $\mu$ is supposed of the form:

$$
\mu=f-\operatorname{div} F,
$$

where $f \in L^{1}(\Omega)$ and $F \in\left(L^{p^{\prime}(x)}\left(\Omega, v^{*}\right)\right)^{N}$.

A typical example of the problem $(\mathcal{P})$ is the following involving the so-called $p(x)$-Laplacian operator with weight:

$$
\Delta_{v, p(x)} u=\operatorname{div}\left(v(x)|\nabla u|^{p(x)-2} \nabla u\right) .
$$

The operator $\Delta_{v, p(x)}$ becomes $p$-Laplacian when $p(x) \equiv p \quad$ (a constant) and $v(x) \equiv 1$. The $p(x)$-Laplacian operator with weight possesses more complicated nonlinearities than the classical $p$-Laplacian, for example, it is inhomogeneous with some degeneracy or singularity. For the applied background of $p(x)$-Laplacian, we refer to (see [1]). The study of differential equations with variable exponents has been a very active field in recent years, we find applications in electro-rheological fluids (see [1] and [2]) and in image processing (see [3]).

Under our assumptions (in particular (1.5), the problem $(\mathcal{P})$ does not admit, in general, a weak solution since the term $a(x, u, \nabla u)$ may not belong to $\left(L_{\text {loc }}^{1}(\Omega)\right)^{N}$. To overcome this difficulty we use in this paper the framework of $L^{1}$-version of Minty's lemma (similar to the one used in [4]). And due to the assumption (1.6) it may be a degenerated or singular problem. Note also that, since the datum is a measure, then the notion of a weak solution cannot be used, 
hence it is replaced by another approach of solution calling $T-v-p(x)$-solution (see definition 3.1 below).

Dirichlet problem of type $(\mathcal{P})$ was considered in ([5] [6]), where in the first work the case of $p(x) \equiv p$ (a constant) and $v(x) \equiv 1$ is treated, while the second work concerns the degenerated case with $p(x) \equiv p$ (a constant). Hence our present paper can be seen as a generalization of the two works ([5] [6]). We also point out that the existence of solutions for elliptic equations with variable exponents can be found in [7] [8] and [9] and.

This paper is divided into three sections, organized as follows: In Section 2, we introduce and prove some properties of the weighted Sobolev spaces with variable exponent and in Section 3, we prove the existence of $T-v-p(x)$-solutions of our problem $(\mathcal{P})$. Among the research objectives of this article is to introduce it for applications in physics and also will be a platform for the problem systems of Dirichlet and others.

\section{Weighted Sobolev Spaces with Variable Exponent}

Let $p \in C_{+}(\bar{\Omega})$ and $v$ be a weighted function in $\Omega$.

We define the weighted Lebesgue space with variable exponents $L^{p(x)}(\Omega, v)$ as the set of all measurable functions $u: \Omega \rightarrow \mathbb{R}$ for which the convex weightmodular

$$
\rho_{v, p(x)}(u)=\int_{\Omega} v(x)|u|^{p(x)} \mathrm{d} x
$$

is finite. The expression

$$
\|u\|_{p(x), v}=\inf \left\{\mu>0: \int_{\Omega} v(x)\left|\frac{u}{\mu}\right|^{p(x)} \mathrm{d} x \leq 1\right\}
$$

defines a norm in $L^{p(x)}(\Omega, v)$, called the Luxemburg norm.

Proposition 2.1. The space $\left(L^{p(x)}(\Omega, v),\|\cdot\|_{p(x), v}\right)$ is a Banach space.

Proof. By considering the operator $M_{v^{\frac{1}{p(x)}}}: L^{p(x)}(\Omega, v) \rightarrow L^{p(x)}(\Omega)$ defined by

$$
M_{v^{\frac{1}{p(x)}}}(f)=f v^{\frac{1}{p(x)}}
$$

for all $f \in L^{p(x)}(\Omega, v)$, it's easy to show that $M_{v_{v^{p(x)}}}$ is an isomorphism and hence we can deduce.

Remark 2.1. When $v(x) \equiv 1$, the weighted Lebesgue spaces with variable exponent $L^{p(x)}(\Omega, v)$ coincides with the Lebesgue space with variable exponent $L^{p(x)}(\Omega)$.

The weight-modular $\rho_{v, p(x)}$ coincides with the modular $\rho_{p(x)}$ defined on $L^{p(x)}(\Omega)$ by $\rho_{p(x)}(u):=\int_{\Omega}|u|^{p(x)} \mathrm{d} x$ (for more details see [10] [11] [12] and [13]).

Lemma 2.1. For all function $u \in L^{p(x)}(\Omega, v)$, the following assertions are sa- 
tisfied:

1) $\rho_{v, p(x)}(u)>1(=1 ;<1) \Leftrightarrow\|u\|_{p(x), v}>1(=1 ;<1)$, respectively.

2) If $\|u\|_{p(x), v}>1$, then $\|u\|_{p(x), v}^{p_{-}} \leq \rho_{v, p(x)}(u) \leq\|u\|_{p(x), v}^{p_{+}}$.

3) If $\|u\|_{p(x), v}<1$, then $\|u\|_{p(x), v}^{p_{+}} \leq \rho_{v, p(x)}(u) \leq\|u\|_{p(x), v}^{p_{-}}$.

Proof. It suffices to remark that $\rho_{v, p(x)}(u)=\rho_{p(x)}\left(v^{\frac{1}{p(x)}} u\right)$ and $\left\|\frac{1}{v^{p(x)}} u\right\|=\|u\|_{p(x), v}$, and using the analogous result in [13].

Proposition 2.2. Let $\Omega$ be a bounded open domain of $\mathbb{R}^{N}$ and $v$ be a weight function on $\Omega$ satifying the integrability condtions (1.1) and (1.2). Then $L^{p(x)}(\Omega, v) \hookrightarrow L_{\text {loc }}^{1}(\Omega)$.

Proof.

Let $K$ be an included compact on $\Omega$. By vertue of Hölder inequality we have,

$$
\begin{aligned}
\int_{K}|u| \mathrm{d} x & =\int_{K}|u|^{\frac{1}{p(x)}} v^{\frac{-1}{p(x)}} \mathrm{d} x \\
& \leq 2\left\||u| v^{\frac{1}{p(x)}}\right\|\left\|_{L^{p(x)}(K)}\right\| \frac{-1}{p(x)}\|\|_{L^{p^{\prime}(x)}(K)} \\
& \leq 2\|u\|_{p(x), v}\left(\int_{K} v^{\frac{-p^{\prime}(x)}{p(x)}} \mathrm{d} x+1\right)^{\frac{1}{p_{-}^{\prime}}} \\
& \leq 2\|u\|_{p(x), v}\left(\int_{K} v^{\frac{-1}{p(x)-1}} \mathrm{~d} x+1\right)^{\frac{1}{p_{-}^{\prime}}} .
\end{aligned}
$$

Hence, the conditions (1.1) and (1.2) allow to conclude.

We define the weighted Sobolev space with variable exponents denoted $W^{1, p(x)}(\Omega, v)$, by

$$
W^{1, p(x)}(\Omega, v)=\left\{u \in L^{p(x)}(\Omega): \frac{\partial u}{\partial x_{i}} \in L^{p(x)}(\Omega, v), i=1, \cdots, N\right\},
$$

equipped with the norm

$$
\|u\|_{1, p(x), v}=\|u\|_{p(x)}+\sum_{i=1}^{N}\left\|\frac{\partial u}{\partial x_{i}}\right\|_{p(x), v}
$$

which is equivalent to the Luxemburg norm

$$
\|\| u \mid \|=\inf \left\{\mu>0: \int_{\Omega}\left(\left|\frac{u}{\mu}\right|^{p(x)}+v(x) \sum_{i=1}^{N}\left|\frac{\frac{\partial u}{\partial x_{i}}}{\mu}\right|^{p(x)}\right) \mathrm{d} x \leq 1\right\} .
$$

Proposition 2.3. Let $v$ be a weight function on $\Omega$ satisfying the conditions (1.1) and (1.2). Then the space $\left(W^{1, p(x)}(\Omega, v),\|\cdot\|_{1, p(x), v}\right)$ is a Banach space. 
Proof. Let $\left(u_{n}\right)_{n}$ be a Cauchy sequence in $\left(W^{1, p(x)}(\Omega, v),\|\cdot\|_{1, p(x), v}\right)$. Then $\left(u_{n}\right)_{n}$ is a Cauchy sequence in $L^{p(x)}(\Omega)$ and $\left(\frac{\partial u_{n}}{\partial x_{i}}\right)_{n}$ is also a Cauchy sequence in $L^{p(x)}(\Omega, v)$ for all $i=1, \cdots, N$. By vertue of proposition 2.1, we can deduce that there exist $u \in L^{p(x)}(\Omega)$ and $v_{i} \in L^{p(x)}(\Omega, v)$ such that:

$$
u_{n} \rightarrow u \text { in } L^{p(x)}(\Omega)
$$

and

$$
\frac{\partial u_{n}}{\partial x_{i}} \rightarrow v_{i} \text { in } L^{p(x)}(\Omega, v) \text { for all } i=1, \cdots, N .
$$

Moreover, by using proposition 2.2, we have $L^{p(x)}(\Omega, v) \subset L_{\text {loc }}^{1}(\Omega) \subset D^{\prime}(\Omega)$. Thus, for all $\varphi \in D(\Omega)$ one has,

$$
\left\langle T_{v_{i}}, \varphi\right\rangle=\lim _{n \rightarrow \infty}\left\langle T_{\frac{\partial u_{n}}{\partial x_{i}}}, \varphi\right\rangle=-\lim _{n \rightarrow \infty}\left\langle T_{u_{n}}, \frac{\partial \varphi}{\partial x_{i}}\right\rangle=-\left\langle T_{u}, \frac{\partial \varphi}{\partial x_{i}}\right\rangle=\left\langle T_{\frac{\partial u}{\partial x_{i}}}, \varphi\right\rangle .
$$

Hence $T_{v_{i}}=T_{\frac{\partial u}{\partial x_{i}}}$, i.e. $v_{i}=\frac{\partial u}{\partial x_{i}}$.

Consequently,

$$
u \in W^{1, p(x)}(\Omega, v)
$$

and

$$
u_{n} \rightarrow u \text { in } W^{1, p(x)}(\Omega, v) .
$$

Remark 2.2. Since $v$ satisfies the conditions (1.1) and (1.2), it's easy to prove that $C_{0}^{\infty}(\Omega)$ is included in $W^{1, p(x)}(\Omega, v)$; then we can define the following space

$$
W_{0}^{1, p(x)}(\Omega, v)=\overline{C_{0}^{\infty}(\Omega)^{\|\cdot\|_{1, p(x), v}},}
$$

which is also a Banach space under the norm $\|\cdot\|_{1, p(x), v}$.

Proposition 2.4. (Characterization of the dual space).

Let $p(.) \in C_{+}(\bar{\Omega})$ and $v$ be a weight function on $\Omega$ satisfying the conditions (1.1) and (1.2). Then for all $G \in\left(W_{0}^{1, p(x)}(\Omega, v)\right)^{*}$, there exists a unique system of functions $\left(g_{0}, g_{1}, \cdots, g_{N}\right) \in L^{p^{\prime}(x)}(\Omega) \times\left(L^{p^{\prime}(x)}\left(\Omega, v^{1-p^{\prime}(x)}\right)\right)^{N}$ such that,

$$
G(f)=\int_{\Omega} f(x) g_{0}(x) \mathrm{d} x+\sum_{i=1}^{N} \int_{\Omega} \frac{\partial f}{\partial x_{i}} g_{i}(x) \mathrm{d} x, \forall f \in W_{0}^{1, p(x)}(\Omega, v) .
$$

Proof. The proof of this proposition is similar to that used in [12] (theorem3.16).

Now, let us introduce the function $p_{s}$ defined by

$$
p_{s}(x)=\frac{p(x) s(x)}{s(x)+1} \text {. }
$$

We have 


$$
p_{s}(x)<p(x) \text { a.e. in } \Omega
$$

and

$$
\left\{\begin{array}{l}
p_{s}^{*}(x)=\frac{N p_{s}(x)}{N-p_{s}(x)}=\frac{N p(x) s(x)}{N(s(x)+1)-p(x) s(x)} \text { if } p(x) s(x)<N(s(x)+1), \\
p_{s}^{*}(x) \text { is arbitrary, otherwise. }
\end{array}\right.
$$

Proposition 2.5. Let $p, s \in C_{+}(\bar{\Omega})$ and $v$ be a weight function on $\Omega$ which satisfies the conditions (1.1), (1.2) and (1.3). Then $W^{1, p(x)}(\Omega, v) \hookrightarrow W^{1, p_{s}(x)}(\Omega)$.

Proof. According to the Hölder inequality and the condition (1.3), one has

$$
\begin{aligned}
\int_{\Omega}|v(x)|^{p_{s}(x)} \mathrm{d} x & =\int_{\Omega}|v(x)|^{p_{s}(x)} v^{\frac{p_{s}(x)}{p(x)}} v^{\frac{-p_{s}(x)}{p(x)}} \mathrm{d} x \\
& \leq\left.\left(\frac{1}{\left(\frac{p}{p_{s}}\right)_{-}}+\frac{1}{(s+1)^{-}}\right)|| v(x)\right|^{p_{s}(x)} v^{\frac{p_{s}(x)}{p(x)}}\left\|_{\frac{p(x)}{p_{s}(x)}} \mid v \frac{-p_{s}(x)}{p(x)}\right\|_{s(x)+1} \\
& \leq\left(\frac{1}{\left(\frac{p}{p_{s}}\right)_{-}}+\frac{1}{(s+1)^{-}}\right)\left(\int_{\Omega}|v(x)|^{p(x)} v(x) \mathrm{d} x\right)^{\frac{1}{\gamma_{1}}}\left(\int_{\Omega} v(x)^{-s(x)} \mathrm{d} x\right)^{\frac{1}{\gamma_{1}}} \\
& \leq C\left(\int_{\Omega}|v(x)|^{p(x)} v(x) \mathrm{d} x\right)^{\frac{1}{\gamma_{1}}}\left(\int_{\Omega} v(x)^{-s(x)} \mathrm{d} x\right)^{\frac{1}{\gamma_{1}}} \\
& \leq C\left(\int_{\Omega}|v(x)|^{p(x)} v(x) \mathrm{d} x\right)^{\frac{1}{\gamma_{1}}} .
\end{aligned}
$$

If we take $v=\frac{\partial u}{\partial x_{i}}$, we then obtain

$$
\int_{\Omega}\left|\frac{\partial u}{\partial x_{i}}\right|^{p_{s}(x)} \mathrm{d} x \leq C\left(\int_{\Omega}\left|\frac{\partial u}{\partial x_{i}}\right|^{p(x)} v(x) \mathrm{d} x\right)^{\frac{1}{\gamma_{1}}}
$$

where

$$
\gamma_{1}= \begin{cases}\left(\frac{p}{p_{s}}\right)_{-} & \text {if }\left\|\left.\frac{\partial u}{\partial x_{i}}(x)\right|^{p_{s}(x)} v^{\frac{p_{s}(x)}{p(x)}}\right\|_{\frac{p(x)}{p_{s}(x)}} \geq 1, \\ \left(\frac{p}{p_{s}}\right)^{+} & \text {if }\left\|\left.\frac{\partial u}{\partial x_{i}}(x)\right|^{p_{s}(x)} v^{\frac{p_{s}(x)}{p(x)}}\right\|_{\frac{p(x)}{p_{s}(x)}}<1 .\end{cases}
$$

Consequently, we can write

$$
\left\|\frac{\partial u}{\partial x_{i}}(x)\right\|_{p_{s}(x)}^{\gamma_{2}} \leq C\left(\int_{\Omega}\left|\frac{\partial u}{\partial x_{i}}\right|^{p(x)} v(x) \mathrm{d} x\right)^{\frac{1}{\gamma_{1}}} \leq C_{0} C_{1}\left\|\frac{\partial u}{\partial x_{i}}(x)\right\|_{p(x), v}^{\gamma_{3}}
$$

where 


$$
\gamma_{2}= \begin{cases}\left(p_{s}\right)_{-} & \text {if }\left\|\frac{\partial u}{\partial x_{i}}(x)\right\|_{p_{s}(x)} \geq 1, \\ \left(p_{s}\right)^{+} & \text {if }\left\|\frac{\partial u}{\partial x_{i}}(x)\right\|_{p_{s}(x)}<1,\end{cases}
$$

and

$$
\gamma_{3}= \begin{cases}p^{+} & \text {si }\left\|\frac{\partial u}{\partial x_{i}}(x)\right\|_{p(x), v} \geq 1, \\ p_{-} & \text {si }\left\|\frac{\partial u}{\partial x_{i}}(x)\right\|_{p(x), v}<1 .\end{cases}
$$

Thus

$$
\left\|\frac{\partial u}{\partial x_{i}}\right\|_{p_{s}(x)} \leq C\left\|\frac{\partial u}{\partial x_{i}}\right\|_{p(x), v}^{\frac{\gamma_{3}}{\gamma_{1} \gamma_{2}}}, i=1,2, \cdots, N .
$$

Note that $C=c\left(\gamma_{1}, \gamma_{2}, \gamma_{3}\right)$ denotes some positive constant which may be changing step by step.

Since $p_{s}(x)<p(x)$ p.p. in $\Omega$, then, there exists a positive constant $C$ such that

$$
\|u\|_{L^{p_{s}(x)}(\Omega)} \leq C\|u\|_{L^{p(x)}(\Omega)} .
$$

Thus, we conclude that

$$
W^{1, p(x)}(\Omega, v) \hookrightarrow W^{1, p_{s}(x)}(\Omega) .
$$

Corollary 2.1. Let $p, s \in C_{+}(\bar{\Omega})$ and $v$ be a weight on $\Omega$ which satisfies the conditions (1.1), (1.2) and (1.3). Then $W^{1, p(x)}(\Omega, v) \hookrightarrow \hookrightarrow L^{r(x)}(\Omega)$, for $1 \leq r(x)<p_{s}^{*}(x)$.

Corollary 2.2. Let $p \in C_{+}(\bar{\Omega})$ and $v$ be a weight function on $\Omega$ which satisfies the conditions (1.1), (1.2) and (1.3). Then

$$
\|u\|_{L^{p(x)}(\Omega)} \leq C\|\nabla u\|_{L^{p(x)}(\Omega ; v)}, \quad \forall u \in \mathcal{C}_{0}^{\infty}(\Omega) .
$$

Proof. Let $u \in \mathcal{C}_{0}^{\infty}(\Omega)$. Since $1 \leq p(x)<p_{s}^{*}(x)$, we deduce by vertue of the embedding $W^{1, p_{s}(x)}(\Omega) \hookrightarrow L^{p(x)}(\Omega)$ that,

$$
\|u\|_{L^{p(x)}(\Omega)} \leq C_{1}\left(\|u\|_{L^{p_{s}(x)}(\Omega)}+\|\nabla u\|_{\left(L^{p_{s}(x)}(\Omega)\right)^{N}}\right) .
$$

Thus, in view of the proposition 2.5 , we obtain

$$
\|u\|_{L^{p(x)}(\Omega)} \leq C_{2}\|\nabla u\|_{L^{p_{S}}(\Omega)} \leq C_{3}\|\nabla u\|_{L^{p(x)}(\Omega ; v)},
$$

which allows to conclude that

$$
\|u\|_{L^{p(x)}(\Omega)} \leq C\|\nabla u\|_{L^{p(x)}(\Omega ; v)} .
$$

\section{Existence Result}

Consider the nonhomogeneous nonlinear Dirichlet boundary problem: 
$(\mathcal{P})\left\{\begin{array}{l}-\operatorname{div}(a(x, u, \nabla u))=-\operatorname{div} F \quad \text { in } \Omega \\ u=0 \quad \text { on } \partial \Omega .\end{array}\right.$

Definition 3.1. A function $u$ is called a $T-v-p(x)$-solution of problem $(\mathcal{P})$ if: $\left\{\begin{array}{l}u \in W_{0}^{1, p(x)}(\Omega, v), \\ \int_{\Omega} a(x, u, \nabla u) \nabla T_{k}(u-\varphi) \mathrm{d} x=\int_{\Omega} f T_{k}(u-\varphi) \mathrm{d} x+\int_{\Omega} F \nabla T_{k}(u-\varphi) \mathrm{d} x, \forall \varphi \in W_{0}^{1, p(x)}(\Omega, v) \cap L^{\infty}(\Omega) .\end{array}\right.$

Theorem 3.1. Let suppose that the assumptions (1.1)-(1.7) are satisfied. Then the problem $(\mathcal{P})$ has at least one $T-v-p(x)$-solution.

Remark 3.1. Note that in the particular case where $p(.) \equiv p$ (constant), $\gamma(r)=1$ and $v=1$, the same result is proved in [14] by using the approach of pseudo-monotonicity.

\subsection{Approximate Problem}

Let $\left(f_{n}\right)_{n}$ be a sequence of functions in $L^{\infty}(\Omega)$ which converges strongly to $f$ in $L^{1}(\Omega)$ such that $\left\|f_{n}\right\|_{L^{\infty}(\Omega)} \leq\|f\|_{L^{\infty}(\Omega)}$. For $n \geq 1$, we consider the approximate problem of $(\mathcal{P})$

$$
\left(\mathcal{P}_{n}\right)\left\{\begin{array}{l}
u_{n} \in W_{0}^{1, p(x)}(\Omega, v) \\
-\operatorname{div}\left(a\left(x, T_{n}\left(u_{n}\right), \nabla u_{n}\right)\right)=f_{n}-\operatorname{div} F \quad \text { in } \Omega .
\end{array}\right.
$$

This section is devoted to establishing the existing solution for the approximate problem $\left(\mathcal{P}_{n}\right)$.

Theorem 3.2. The operator $A_{k}$ defined by,

$$
\begin{aligned}
& A_{k}: W_{0}^{1, p(x)}(\Omega, v) \rightarrow W^{-1, p^{\prime}(x)}\left(\Omega, v^{*}\right) \\
& u \mapsto A_{k} u=-\operatorname{div}\left(a\left(x, T_{k}(u), \nabla u\right)\right)
\end{aligned}
$$

is bounded, coercive, hemicontinuous and pseudo-monotone.

\section{Proof of Theorem 3.2}

- The operator $A_{k}$ is bounded. Indeed for all $u, v \in W_{0}^{1, p(x)}(\Omega, v)$, one has

$$
\begin{aligned}
& \left|\left\langle A_{k} u, v\right\rangle\right|=\left|\int_{\Omega} a\left(x, T_{k}(u), \nabla u\right) \nabla v \mathrm{~d} x\right|=\left|\int_{\Omega} a\left(x, T_{k}(u), \nabla u\right) v^{\frac{-1}{p(x)}} \nabla v v^{\frac{1}{p(x)}} \mathrm{d} x\right| \\
& \leq\left.\left(\frac{1}{p_{-}}+\frac{1}{p_{-}^{\prime}}\right)|| a\left(x, T_{k}(u), \nabla u\right) v^{\frac{-1}{p(x)}}\right|_{L^{p^{\prime}(x)}(\Omega)} \mid \nabla v v^{\frac{1}{p(x)}}\|\|_{L^{p(x)}(\Omega)} \\
& \leq 2\left(\left.\int_{\Omega}\left|a\left(x, T_{k}(u), \nabla u\right) v^{\frac{-1}{p(x)}}\right|^{p^{\prime}(x)} \mathrm{d} x\right|^{\frac{1}{p_{-}^{\prime}}}\|\nabla v\|_{L^{p(x)}(\Omega, v)}\left(\int_{\Omega}\left(b(x)+\left|T_{k}(u)\right|^{p(x)-1}+v^{\frac{1}{p^{\prime}(x)}}\left(\gamma\left(T_{k}(u)\right)|\nabla u|\right)^{p(x)-1}\right)^{p^{\prime}(x)} \mathrm{d} x\right)^{\frac{1}{p_{-}^{\prime}}}\|\nabla v\|_{L^{p(x)}(\Omega, v)}\right. \\
& \leq C_{1}\left(\int_{\Omega}\left(b(x)^{p^{\prime}(x)}+\left|T_{k}(u)\right|^{p(x)}+v(x)\left(\gamma\left(T_{k}(u)\right)|\nabla u|\right)^{p(x)}\right) \mathrm{d} x\right)^{\frac{1}{p_{-}^{\prime}}}\|v\|_{W_{0}^{1, p(x)}(\Omega, v)}
\end{aligned}
$$




$$
\leq\left(C_{1}+C_{2}+C_{3}\left(\int_{\Omega}\left|T_{k}(u)\right|^{p(x)}+v(x)\left(\gamma\left(T_{k}(u)\right)|\nabla u|\right)^{p(x)} \mathrm{d} x\right)^{\frac{1}{p_{-}^{\prime}}}\right)\|v\|_{w_{0}^{1, p(x)}(\Omega, v)} .
$$

Since $\gamma($.$) is continuous and \left|T_{k}(u)\right| \leq k$ a.e. in $\Omega$, then $\gamma\left(T_{k}(u)\right)|\nabla u|$ is bounded in $W_{0}^{1, p(x)}(\Omega, v)$; hence the operator $A_{k}$ is bounded.

- The operator $A_{k}$ is hemicontinuous. Indeed, let $t$ be a reality that tends to $t_{0}$. We have

$$
a\left(x, T_{k}(u+t v), \nabla T_{k}(u+t v)\right) \rightarrow a\left(x, T_{k}\left(u+t_{0} v\right), \nabla T_{k}\left(u+t_{0} v\right)\right) \text {, a.e. in } \Omega .
$$

Since $\left(a\left(x, T_{k}(u+t v), \nabla T_{k}(u+t v)\right)\right)$ is bounded in $\left(L^{p^{\prime}}(\Omega)\right)^{N}$, we deduce that $A_{k}(u+t v)$ converges to $A_{k}\left(u+t_{0} v\right)$ weakly in $W^{-1, p^{\prime}(x)}\left(\Omega, v^{*}\right)$ as $t$ tends to $t_{0}$.

- The operator $A_{k}$ is coercive. Indeed, for all $u \in W_{0}^{1, p(x)}(\Omega, v)$, we have

$$
\frac{\left\langle A_{k} u, u\right\rangle}{\|u\|_{W_{0}^{1, p(x)}(\Omega, v)}} \geq \frac{\int_{\Omega} v(x)|\nabla u|^{p(x)} \mathrm{d} x}{\|u\|_{W_{0}^{1, p(x)}(\Omega, v)}} \geq \frac{\|u\|_{W_{0}^{1, p(x)}(\Omega, v)}^{\delta}}{\|u\|_{W_{0}^{1, p(x)}(\Omega, v)}} \geq\|u\|_{W_{0}^{1, p(x)}(\Omega, v)}^{\delta-1},
$$

where

$$
\delta= \begin{cases}p_{-} & \text {if }\|u\|_{W_{0}^{1, p(x)}(\Omega, v)} \leq 1, \\ p^{+} & \text {if }\|u\|_{W_{0}^{1, p(x)}(\Omega, v)}>1,\end{cases}
$$

Obviously, we have $\|u\|_{W_{0}^{1, p(x)}(\Omega, v)}^{\delta-1}$ tends to infinity, when $\|u\|_{W_{0}^{1, p(x)}(\Omega, v)} \rightarrow \infty$, hence we conclude.

- It remains to show that $A_{k}$ is pseudo-monotone: Let $\left(u_{j}\right)_{j}$ be a sequence in $W_{0}^{1, p(x)}(\Omega, v)$ such that

$$
u_{j} \rightarrow u \text { in } W_{0}^{1, p(x)}(\Omega, v) \text { and } \limsup _{j}\left\langle A_{k} u_{j}, u_{j}-u\right\rangle \leq 0 .
$$

Firstly, we prove that $A_{k} u_{j}$ converges to $A_{k} u$ weakly in $W^{-1, p^{\prime}(x)}\left(\Omega, v^{*}\right)$. Indeed, since $\left(u_{j}\right)_{j}$ is a bounded sequence in $W_{0}^{1, p(x)}(\Omega, v)$, then by the growth condition, $\left(A_{k} u_{j}\right)_{j}$ is bounded in $W^{-1, p^{\prime}(x)}\left(\Omega, v^{*}\right)$, therefore there exists a function $h_{k}=\left(h_{k i}\right)$ such that,

$$
\begin{aligned}
& A_{k} u_{j} \rightarrow h_{k} \text { dans } W^{-1, p^{\prime}(x)}\left(\Omega, v^{*}\right), \\
& a_{i}\left(x, T_{k}\left(u_{j}\right), \nabla u_{j}\right) \rightarrow h_{k i} \text { in } L^{p^{\prime}(x)}\left(\Omega, v^{*}\right), \text { for } i=1, \cdots, N .
\end{aligned}
$$

Hence, we can write

$$
\underset{j}{\limsup }\left\langle A_{k} u_{j}, u_{j}\right\rangle \leq\left\langle h_{k}, u\right\rangle .
$$

On the one hand, by (1.5), we have

$$
\begin{aligned}
& \sum_{i=1}^{N} \int_{\Omega}\left(a_{i}\left(x, T_{k}\left(u_{j}\right), \nabla v\right)-a_{i}\left(x, T_{k}\left(u_{j}\right), \nabla u_{j}\right)\right)\left(\frac{\partial v}{\partial x_{i}}-\frac{\partial u_{j}}{\partial x_{i}}\right) \mathrm{d} x \geq 0, \\
& \forall v \in W_{0}^{1, p(x)}(\Omega, v) .
\end{aligned}
$$

Then 


$$
\begin{aligned}
& \sum_{i=1}^{N} \int_{\Omega} a_{i}\left(x, T_{k}\left(u_{j}\right), \nabla u_{j}\right) \frac{\partial u_{j}}{\partial x_{i}} \mathrm{~d} x \\
& \geq \sum_{i=1}^{N} \int_{\Omega} a_{i}\left(x, T_{k}\left(u_{j}\right), \nabla u_{j}\right) \frac{\partial v}{\partial x_{i}} \mathrm{~d} x-\sum_{i=1}^{N} \int_{\Omega} a_{i}\left(x, T_{k}\left(u_{j}\right), \nabla v\right) \frac{\partial v}{\partial x_{i}} \mathrm{~d} x \\
& \quad+\sum_{i=1}^{N} \int_{\Omega} a_{i}\left(x, T_{k}\left(u_{j}\right), \nabla v\right) \frac{\partial u_{j}}{\partial x_{i}} \mathrm{~d} x .
\end{aligned}
$$

Since $u_{j} \rightarrow u$ strongly in $L^{p(x)}(\Omega)$ and a.e. in $\Omega$, then $a_{i}\left(x, T_{k}\left(u_{j}\right), \nabla v\right) \rightarrow a_{i}\left(x, T_{k}(u), \nabla v\right)$ strongly in $L^{p^{\prime}(x)}\left(\Omega, v^{*}\right)$ for $i=1, \cdots, N$.

Therefore,

$$
\sum_{i=1}^{N} \int_{\Omega} a_{i}\left(x, T_{k}\left(u_{j}\right), \nabla v\right) \frac{\partial v}{\partial x_{i}} \mathrm{~d} x \rightarrow \sum_{i=1}^{N} \int_{\Omega} a_{i}\left(x, T_{k}(u), \nabla v\right) \frac{\partial v}{\partial x_{i}} \mathrm{~d} x
$$

and

$$
\sum_{i=1}^{N} \int_{\Omega} a_{i}\left(x, T_{k}\left(u_{j}\right), \nabla v\right) \frac{\partial u_{j}}{\partial x_{i}} \mathrm{~d} x \rightarrow \sum_{i=1}^{N} \int_{\Omega} a_{i}\left(x, T_{k}(u), \nabla v\right) \frac{\partial u}{\partial x_{i}} \mathrm{~d} x .
$$

By vertue of (3.2), we have

$$
\sum_{i=1}^{N} \int_{\Omega} a_{i}\left(x, T_{k}\left(u_{j}\right), \nabla u_{j}\right) \frac{\partial v}{\partial x_{i}} \mathrm{~d} x \rightarrow \sum_{i=1}^{N} \int_{\Omega} h_{k i} \frac{\partial v}{\partial x_{i}} \mathrm{~d} x .
$$

Now, combining (3.4)-(3.6) and (3.7), we obtain

$$
\begin{aligned}
& \lim _{j \rightarrow \infty} \sum_{i=1}^{N} \int_{\Omega} a_{i}\left(x, T_{k}\left(u_{j}\right), \nabla u_{j}\right) \frac{\partial u_{j}}{\partial x_{i}} \mathrm{~d} x \\
& \geq \sum_{i=1}^{N} \int_{\Omega} h_{k i} \frac{\partial v}{\partial x_{i}} \mathrm{~d} x+\sum_{i=1}^{N} \int_{\Omega} a_{i}\left(x, T_{k}(u), \nabla v\right) \frac{\partial u}{\partial x_{i}} \mathrm{~d} x \\
& \quad-\sum_{i=1}^{N} \int_{\Omega} a_{i}\left(x, T_{k}(u), \nabla v\right) \frac{\partial v}{\partial x_{i}} \mathrm{~d} x .
\end{aligned}
$$

Due to (3.3), we deduce that

$$
\begin{aligned}
\sum_{i=1}^{N} \int_{\Omega} h_{k i} \frac{\partial u}{\partial x_{i}} \mathrm{~d} x & \geq \sum_{i=1}^{N} \int_{\Omega} h_{k i} \frac{\partial v}{\partial x_{i}} \mathrm{~d} x+\sum_{i=1}^{N} \int_{\Omega} a_{i}\left(x, T_{k}(u), \nabla v\right) \frac{\partial u}{\partial x_{i}} \mathrm{~d} x \\
& -\sum_{i=1}^{N} \int_{\Omega} a_{i}\left(x, T_{k}(u), \nabla v\right) \frac{\partial v}{\partial x_{i}} \mathrm{~d} x .
\end{aligned}
$$

This implies that,

$$
\sum_{i=1}^{N} \int_{\Omega}\left(a_{i}\left(x, T_{k}\left(u_{j}\right), \nabla v\right)-h_{k i}\right)\left(\frac{\partial v}{\partial x_{i}}-\frac{\partial u_{j}}{\partial x_{i}}\right) \mathrm{d} x \geq 0, \quad \forall v \in W_{0}^{1, p(x)}(\Omega, v) .
$$

On the other hand, choose $v=u+t w$ in (3.9) (with $t \in]-1,1[$ ). It's easy to see that

$$
\left.\int_{\Omega}\left(a\left(x, T_{k}(u), \nabla(u+t w)\right)-h_{k}\right) \nabla w \mathrm{~d} x=0, \quad \forall w \in W_{0}^{1, p(x)}(\Omega, v), \forall t \in\right]-1,1[.
$$

Hence $A_{k} u=h_{k} \in W^{-1, p^{\prime}(x)}\left(\Omega, v^{*}\right)$, and we deduce that $A_{k} u_{j}$ weakly converges to $A_{k} u$ in $W^{-1, p^{\prime}(x)}\left(\Omega, v^{*}\right)$.

Secondly, we prove that $\left\langle A_{k} u_{j}, u_{j}\right\rangle \rightarrow\left\langle A_{k} u, u\right\rangle$. Indeed, in view of (3.2) and (3.3), we have 


$$
\limsup \left\langle A_{k} u_{j}, u_{j}\right\rangle \leq\left\langle A_{k} u, u\right\rangle=\left\langle h_{k}, u\right\rangle .
$$

It remains to show that,

$$
\liminf \left\langle A_{k} u_{j}, u_{j}\right\rangle \geq\left\langle A_{k} u, u\right\rangle=\left\langle h_{k}, u\right\rangle .
$$

For that, we have

$$
\begin{aligned}
\left\langle A_{k} u_{j}, u_{j}\right\rangle= & \sum_{i=1}^{N} \int_{\Omega} a_{i}\left(x, T_{k}\left(u_{j}\right), \nabla u_{j}\right) \frac{\partial u_{j}}{\partial x_{i}} \mathrm{~d} x \\
= & \sum_{i=1}^{N} \int_{\Omega}\left(a_{i}\left(x, T_{k}\left(u_{j}\right), \nabla u_{j}\right)-a_{i}\left(x, T_{k}\left(u_{j}\right), \nabla u\right)\right)\left(\frac{\partial u_{j}}{\partial x_{i}}-\frac{\partial u}{\partial x_{i}}\right) \mathrm{d} x \\
& +\sum_{i=1}^{N} \int_{\Omega} a_{i}\left(x, T_{k}\left(u_{j}\right), \nabla u\right)\left(\frac{\partial u_{j}}{\partial x_{i}}-\frac{\partial u}{\partial x_{i}}\right) \mathrm{d} x \\
& +\sum_{i=1}^{N} \int_{\Omega} a_{i}\left(x, T_{k}\left(u_{j}\right), \nabla u_{j}\right) \frac{\partial u}{\partial x_{i}} \mathrm{~d} x .
\end{aligned}
$$

Since $\quad \sum_{i=1}^{N} \int_{\Omega}\left(a_{i}\left(x, T_{k}\left(u_{j}\right), \nabla u_{j}\right)-a_{i}\left(x, T_{k}\left(u_{j}\right), \nabla u\right)\right)\left(\frac{\partial u_{j}}{\partial x_{i}}-\frac{\partial u}{\partial x_{i}}\right) \mathrm{d} x \geq 0 \quad, \quad$ we deduce that

$$
\begin{aligned}
\left\langle A_{k} u_{j}, u_{j}\right\rangle \geq & \left.\sum_{i=1}^{N} \int_{\Omega} a_{i}\left(x, T_{k}\left(u_{j}\right), \nabla u\right)\right)\left(\frac{\partial u_{j}}{\partial x_{i}}-\frac{\partial u}{\partial x_{i}}\right) \mathrm{d} x \\
& +\sum_{i=1}^{N} \int_{\Omega} a_{i}\left(x, T_{k}\left(u_{j}\right), \nabla u_{j}\right) \frac{\partial u}{\partial x_{i}} \mathrm{~d} x .
\end{aligned}
$$

Therefore,

$$
\begin{aligned}
\liminf \left\langle A_{k} u_{j}, u_{j}\right\rangle \geq & \liminf \sum_{i=1}^{N} \int_{\Omega} a_{i}\left(x, T_{k}\left(u_{j}\right), \nabla u\right)\left(\frac{\partial u_{j}}{\partial x_{i}}-\frac{\partial u}{\partial x_{i}}\right) \mathrm{d} x \\
& +\liminf \sum_{i=1}^{N} \int_{\Omega} a_{i}\left(x, T_{k}\left(u_{j}\right), \nabla u_{j}\right) \frac{\partial u}{\partial x_{i}} \mathrm{~d} x .
\end{aligned}
$$

Hence, $\liminf \left\langle A_{k} u_{j}, u_{j}\right\rangle \geq \sum_{i=1}^{N} \int_{\Omega} h_{i} \frac{\partial u}{\partial x_{i}} \mathrm{~d} x \geq\left\langle A_{k} u, u\right\rangle$. This achieved the proof.

\subsection{Proof of Theorem 3.1}

The proof is divided into 4 steps.

Step 1: We will show that $\left(u_{n}\right)_{n}$ is a Cauchy sequence in measure. Using $T_{k}\left(u_{n}\right)$ as a test function in $\left(\mathcal{P}_{n}\right)$ leads to,

$$
\int_{\Omega} a\left(x, T_{k}\left(u_{n}\right), \nabla u_{n}\right) \nabla T_{k}\left(u_{n}\right) \mathrm{d} x=\int_{\Omega} f_{n} T_{k}\left(u_{n}\right) \mathrm{d} x+\int_{\Omega} F \cdot \nabla T_{k}\left(u_{n}\right) \mathrm{d} x .
$$

From (1.6) and (1.7), we deduce for all $k>1$ that,

$$
\begin{aligned}
& \alpha \sum_{i=1}^{N} \int_{\Omega}\left|\frac{\partial T_{k}\left(u_{n}\right)}{\partial x_{i}}\right|^{p(x)} v(x) \mathrm{d} x \\
& \leq k\|f\|_{L^{1}}+\sum_{i=1}^{N} \int_{\Omega}\left|F_{i}\right| v(x)^{\frac{-1}{p(x)}}\left|\frac{\partial T_{k}\left(u_{n}\right)}{\partial x_{i}}\right| v(x)^{\frac{1}{p(x)}} \mathrm{d} x \\
& \leq k\|f\|_{L^{1}}+\sum_{i=1}^{N} \int_{\Omega}\left|F_{i}\right| v(x)^{\frac{-1}{p(x)}}\left(\frac{\alpha}{2}\right)^{\frac{-1}{p(x)}}\left|\frac{\partial T_{k}\left(u_{n}\right)}{\partial x_{i}}\right| v(x)^{\frac{1}{p(x)}}\left(\frac{\alpha}{2}\right)^{\frac{1}{p(x)}} \mathrm{d} x .
\end{aligned}
$$


Now, by Young's inequality, we obtain

$$
\begin{aligned}
& \alpha \sum_{i=1}^{N} \int_{\Omega}\left|\frac{\partial T_{k}\left(u_{n}\right)}{\partial x_{i}}\right|^{p(x)} v(x) \mathrm{d} x \\
& \leq k\|f\|_{L^{1}}+\sum_{i=1}^{N} \int_{\Omega}\left|F_{i}\right|^{p^{\prime}(x)} v(x)^{\frac{-p^{\prime}(x)}{p(x)}} \frac{C(\alpha)}{p^{\prime}(x)} \mathrm{d} x+\sum_{i=1}^{N} \int_{\Omega}\left|\frac{\partial T_{k}\left(u_{n}\right)}{\partial x_{i}}\right|^{p(x)} v(x) \frac{\alpha}{2 p(x)} \mathrm{d} x \\
& \leq k\|f\|_{L^{1}}+\sum_{i=1}^{N} \int_{\Omega}\left|F_{i}\right|^{p^{\prime}(x)} v(x)^{\frac{-p^{\prime}(x)}{p(x)}} C\left(\alpha, p^{\prime-}\right) \mathrm{d} x+\sum_{i=1}^{N} \int_{\Omega}\left|\frac{\partial T_{k}\left(u_{n}\right)}{\partial x_{i}}\right|^{p(x)} v(x) \frac{\alpha}{2 p^{-}} \mathrm{d} x .
\end{aligned}
$$

Then, one has

$$
\begin{aligned}
& \left(1-\frac{1}{2 p^{-}}\right) \alpha \sum_{i=1}^{N} \int_{\Omega}\left|\frac{\partial T_{k}\left(u_{n}\right)}{\partial x_{i}}\right|^{p(x)} v(x) \mathrm{d} x \\
& \leq k\|f\|_{L^{1}}+\frac{C\left(\alpha, p^{\prime-}\right)}{k}+\sum_{i=1}^{N} \int_{\Omega}\left|F_{i}\right|^{p^{\prime}(x)} v(x)^{\frac{-p^{\prime}(x)}{p(x)}} \mathrm{d} x,
\end{aligned}
$$

for $k \geq 1$, which implies that

$$
\sum_{i=1}^{N} \int_{\Omega}\left|\frac{\partial T_{k}\left(u_{n}\right)}{\partial x_{i}}\right|^{p(x)} v(x) \mathrm{d} x \leq C k \text { for all } k>1 .
$$

Let $k>0$ large enough and $B_{R}$ be a ball of $\Omega$. Using (3.11) and applying Hölder's inequality and Poincaré's inequality, we obtain

$$
\begin{gathered}
\quad k \text { meas }\left(\left\{\left|u_{n}\right|>k\right\} \cap B_{R}\right) \\
=\int_{\left\{\left|u_{n}\right|>k\right\} \cap B_{R}}\left|T_{k}\left(u_{n}\right)\right| \mathrm{d} x \leq\left\|T_{k}\left(u_{n}\right)\right\|_{L^{1}(\Omega)} \leq C\left\|T_{k}\left(u_{n}\right)\right\|_{L^{p(x)}(\Omega)} \\
\leq C\left\|\nabla T_{k}\left(u_{n}\right)\right\|_{p(x), v} \quad \text { by vertue of Corollary 2.2) } \\
\leq C\left(\int_{\Omega} \sum_{i=1}^{N}\left|\frac{\partial T_{k}\left(u_{n}\right)}{\partial x_{i}}\right|^{p(x)} v(x) \mathrm{d} x\right)^{\frac{1}{\kappa}} \quad \text { by vertue of Lemma 2.1) } \\
\leq C k^{\frac{1}{\kappa}},
\end{gathered}
$$

where

$$
\kappa= \begin{cases}p_{-} & \text {if }\left\|\nabla T_{k}\left(u_{n}\right)\right\|_{p(x), v} \leq 1, \\ p_{+} & \text {if }\left\|\nabla T_{k}\left(u_{n}\right)\right\|_{p(x), v}>1,\end{cases}
$$

which implies that,

$$
\operatorname{meas}\left(\left\{\left|u_{n}\right|>k\right\} \cap B_{R}\right) \leq \frac{C}{k^{1-\frac{1}{\kappa}}}, \forall k>1 .
$$

So, we have, for all $\delta>0$,

$$
\begin{aligned}
& \text { meas }\left(\left\{\left|u_{n}-u_{m}\right|>\delta\right\} \cap B_{R}\right) \\
& \leq \text { meas }\left(\left\{\left|u_{n}\right|>k\right\} \cap B_{R}\right)+\text { meas }\left(\left\{\left|u_{m}\right|>k\right\} \cap B_{R}\right) \\
& \quad+\text { meas }\left(\left\{\left|T_{k}\left(u_{n}\right)-T_{k}\left(u_{m}\right)\right|>\delta\right\}\right) .
\end{aligned}
$$


Since $\left(T_{k}\left(u_{n}\right)\right)_{n}$ is bounded in $W_{0}^{1, p(x)}(\Omega, v)$, there exists a subsequence, still denoted by $T_{k}\left(u_{n}\right)$ and a measurable function $v_{k} \in W_{0}^{1, p(x)}(\Omega, v)$ such that $T_{k}\left(u_{n}\right)$ converges to $v_{k}$ weakly in $W_{0}^{1, p(x)}(\Omega, v)$, strongly in $L^{p(x)}(\Omega)$ and almost everywhere in $\Omega$. Hence $\left(T_{k}\left(u_{n}\right)\right)_{n}$ is a Cauchy sequence in measure in $\Omega$.

Let $\varepsilon>0$. Then by (3.13), there exists $k(\varepsilon)>0$ such that,

$$
\text { meas }\left(\left\{\left|u_{n}-u_{m}\right|>\delta\right\} \cap B_{R}\right)<\varepsilon, \forall n, m \geq n_{0}(k(\varepsilon), \delta, R) .
$$

This proves that $\left(u_{n}\right)_{n}$ is a Cauchy sequence in measure in $B_{R}$, thus converges almost everywhere to some measurable function $u$. Hence

$$
\begin{aligned}
& T_{k}\left(u_{n}\right) \rightarrow T_{k}(u) \text { weakly in } W_{0}^{1, p(x)}(\Omega, v), \\
& \text { strongly in } W^{p(x)}(\Omega) \text {, and a.e. in } \Omega \text {. }
\end{aligned}
$$

Step 2: We shall prove that

$$
\begin{aligned}
& \int_{\Omega} a\left(x, u_{n}, \nabla \varphi\right) \nabla T_{k}\left(u_{n}-\varphi\right) \mathrm{d} x \\
& \leq \int_{\Omega} f_{n} T_{k}\left(u_{n}-\varphi\right) \mathrm{d} x+\int_{\Omega} F \nabla T_{k}\left(u_{n}-\varphi\right) \mathrm{d} x \\
& \forall \varphi \in W_{0}^{1, p(x)}(\Omega, v) \cap L^{\infty}(\Omega) .
\end{aligned}
$$

Let $\varphi \in W_{0}^{1, p(x)}(\Omega, v) \cap L^{\infty}(\Omega)$ and let $n$ be large enough $\left(n \geq k+\|\varphi\|_{\infty}\right)$. Using the admissible test function $T_{k}\left(u_{n}-\varphi\right)$ in $\left(\mathcal{P}_{n}\right)$ leads to

$$
\begin{aligned}
& \int_{\Omega} a\left(x, u_{n}, \nabla u_{n}\right) \nabla\left(T_{k}\left(u_{n}-\varphi\right)\right) \mathrm{d} x=\int_{\Omega} f_{n} T_{k}\left(u_{n}-\varphi\right) \mathrm{d} x+\int_{\Omega} F \nabla T_{k}\left(u_{n}-\varphi\right) \mathrm{d} x,(3.17) \\
& \text { i.e., } \\
& \int_{\Omega} a\left(x, u_{n}, \nabla u_{n}\right) \nabla T_{k}\left(u_{n}-\varphi\right) \mathrm{d} x+\int_{\Omega} a\left(x, u_{n}, \nabla \varphi\right) \nabla T_{k}\left(u_{n}-\varphi\right) \mathrm{d} x \\
& -\int_{\Omega} a\left(x, u_{n}, \nabla \varphi\right) \nabla T_{k}\left(u_{n}-\varphi\right) \mathrm{d} x=\int_{\Omega} f_{n} T_{k}\left(u_{n}-\varphi\right) \mathrm{d} x+\int_{\Omega} F \nabla T_{k}\left(u_{n}-\varphi\right) \mathrm{d} x,
\end{aligned}
$$

which implies that

$$
\begin{aligned}
& \int_{\Omega}\left(a\left(x, u_{n}, \nabla u_{n}\right)-a\left(x, u_{n}, \nabla \varphi\right)\right) \nabla T_{k}\left(u_{n}-\varphi\right) \mathrm{d} x \\
& +\int_{\Omega} a\left(x, u_{n}, \nabla \varphi\right) \nabla T_{k}\left(u_{n}-\varphi\right) \mathrm{d} x=\int_{\Omega} f_{n} T_{k}\left(u_{n}-\varphi\right) \mathrm{d} x+\int_{\Omega} F \nabla T_{k}\left(u_{n}-\varphi\right) \mathrm{d} x .
\end{aligned}
$$

Thanks to assumption (1.5) and the definition of truncation function, we have

$$
\int_{\Omega}\left(a\left(x, u_{n}, \nabla u_{n}\right)-a\left(x, u_{n}, \nabla \varphi\right)\right) \nabla T_{k}\left(u_{n}-\varphi\right) \mathrm{d} x \geq 0 .
$$

Combining (3.19) and (3.20), we obtain (3.16).

Step 3: We claim that

$$
\begin{aligned}
& \int_{\Omega} a(x, u, \nabla \varphi) \nabla T_{k}(u-\varphi) \mathrm{d} x \\
& \leq \int_{\Omega} f T_{k}(u-\varphi) \mathrm{d} x+\int_{\Omega} F \nabla T_{k}(u-\varphi) \mathrm{d} x \quad \forall \varphi \in W_{0}^{1, p(x)}(\Omega, v) \cap L^{\infty}(\Omega) .
\end{aligned}
$$

Let $M=k+\|\varphi\|_{\infty}$. Since $T_{M}\left(u_{n}\right)$ converges to $T_{M}(u)$ weakly in $W_{0}^{1, p(x)}(\Omega, v)$, then

$$
T_{k}\left(u_{n}-\varphi\right) \rightarrow T_{k}(u-\varphi) \quad \text { weakly in } W_{0}^{1, p(x)}(\Omega, v) .
$$

Thanks to assumption (1.4), we have 


$$
\begin{aligned}
& \left|a\left(x, T_{M}\left(u_{n}\right), \nabla \varphi\right)\right|^{p^{\prime}(x)} v^{\frac{p^{\prime}(x)}{p(x)}} \\
& \leq \beta\left[b(x)+\left|T_{M}\left(u_{n}\right)\right|^{p(x)-1}+v^{\frac{1}{p^{\prime}(x)}}\left(\gamma\left(T_{M}\left(u_{n}\right)\right)|\nabla \varphi|\right)^{p(x)-1}\right]^{p^{\prime}(x)} \\
& \leq C\left[b(x)^{p^{\prime}(x)}+\left|T_{M}\left(u_{n}\right)\right|^{p(x)}+v(x) \gamma_{0}^{p(x)}|\nabla \varphi|^{p(x)}\right],
\end{aligned}
$$

where $\gamma_{0}=\sup \left\{|\gamma(s)|:|s| \leq k+\|\varphi\|_{\infty}\right\}$ and $C$ is a positive constant. Since $T_{M}\left(u_{n}\right)$ converges to $T_{M}(u)$ weakly in $W_{0}^{1, p(x)}(\Omega, v)$, strongly in $L^{p(x)}(\Omega)$ and a.e. in $\Omega$, thus

$$
\left|a\left(x, T_{M}\left(u_{n}\right), \nabla \varphi\right)\right|^{p^{\prime}(x)} v^{\frac{p^{\prime}(x)}{p(x)}} \rightarrow\left|a\left(x, T_{M}(u), \nabla \varphi\right)\right|^{p^{\prime}(x)} v^{\frac{p^{\prime}(x)}{p(x)}} \text { a.e in } \Omega
$$

and

$$
\begin{aligned}
& C\left[b(x)^{p^{\prime}(x)}+\left|T_{M}\left(u_{n}\right)\right|^{p(x)}+v(x) \gamma_{0}^{p(x)}|\nabla \varphi|^{p(x)}\right] \\
& \rightarrow C\left[b(x)^{p^{\prime}(x)}+\left|T_{M}(u)\right|^{p(x)}+v(x) \gamma_{0}^{p(x)}|\nabla \varphi|^{p(x)}\right] .
\end{aligned}
$$

Combining (3.21), (3.22) and using Vitali's theorem, we obtain

$$
\int_{\Omega} a\left(x, u_{n}, \nabla \varphi\right) \nabla T_{k}\left(u_{n}-\varphi\right) \mathrm{d} x \rightarrow \int_{\Omega} a(x, u, \nabla \varphi) \nabla T_{k}(u-\varphi) \mathrm{d} x .
$$

Now, we show that

$$
\int_{\Omega} f_{n} T_{k}\left(u_{n}-\varphi\right) \mathrm{d} x \rightarrow \int_{\Omega} f T_{k}(u-\varphi) \mathrm{d} x .
$$

In the first time, we have $f_{n} T_{k}\left(u_{n}-\varphi\right) \rightarrow f T_{k}(u-\varphi)$ a.e in $\Omega$, $\left|f_{n} T_{k}\left(u_{n}-\varphi\right)\right| \leq k\left|f_{n}\right|$ and $k\left|f_{n}\right| \rightarrow k|f|$ in $L^{1}(\Omega)$. In the second time, by using Vitali's theorem we obtain (3.25).

Since $F \in\left(L^{p^{\prime}(x)}\left(\Omega, v^{*}\right)\right)^{N}$, one has

$$
\int_{\Omega} F \nabla T_{k}\left(u_{n}-\varphi\right) \mathrm{d} x \rightarrow \int_{\Omega} F \nabla T_{k}(u-\varphi) \mathrm{d} x .
$$

Thanks to (3.24), (3.25) and (3.26), we obtain (3.21).

Step 4: In this step, we introduce the following generalization of Minty's lemma in weighted Sobolev space with variable exponents $W^{1, p(x)}(\Omega, v)$ (which is proved in [15]).

Lemma 3.1. ([15]) Let $u$ be a measurable function such that $T_{k}(u) \in W_{0}^{1, p(x)}(\Omega, v)$ for every $k>0$. Then the following statements are equivalent.

1) $\int_{\Omega} a(x, u, \nabla \varphi) \nabla T_{k}(u-\varphi) \mathrm{d} x \leq \int_{\Omega} f T_{k}(u-\varphi) \mathrm{d} x+\int_{\Omega} F \nabla T_{k}(u-\varphi) \mathrm{d} x$,

2) $\int_{\Omega} a(x, u, \nabla u) \nabla T_{k}(u-\varphi) \mathrm{d} x=\int_{\Omega} f T_{k}(u-\varphi) \mathrm{d} x+\int_{\Omega} F \nabla T_{k}(u-\varphi) \mathrm{d} x$, for every $\varphi \in W_{0}^{1, p(x)}(\Omega, v) \cap L^{\infty}(\Omega)$ and for every $k>0$.

Finally, the result (3.21) and the lemma 3.1 lead to the completion of the proof of theorem 3.1.

\section{Conclusion}

In this article, we have demonstrated the existence of a solution of a problem 
with a second measure member and in the space of Sobolev with variable exponent using Minty's lemma. It is a very important technique in which we use the notions of hemicontinuous and pseudo-monotonic instead of broad or strict monotony.

\section{Conflicts of Interest}

The authors declare no conflicts of interest regarding the publication of this paper.

\section{References}

[1] Ružička, M. (2000) Electrorheological Fluids: Modeling and Mathematical Theory (Lecture Notes in Mathematics, Vol. 1748). Springer, Berlin.

[2] Zhikov, V.V. (2004) On the Density of Smooth Functions in Sobolev-Orlicz Spaces. Zapiski Nauchnykh Seminarov POMI, 310, 67-81.

[3] Chen, Y., Levine, S. and Rao, M. (2006) Variable Exponent, Linear Growth Functionals in Image Restoration. SIAM Journal on Applied Mathematics, 66, 1383-1406. https://doi.org/10.1137/050624522

[4] Boccardo, L. and Orsina. L. (1999) Existence Resultats for Direchlet Problem in $L^{1}$ via Minty's Lemma. Applicable Analysis, 76, 309-313.

https://doi.org/10.1080/00036810008840887

[5] Boccardo, L. (2002) A Remark on Some Nonlinear Elliptic Problem. Electronic Journal of Differential Equations, 8, 47-52.

[6] Akdim, Y., Azroul, E. and Rhoudaf, M. (2008) Existence of T-Solution for Degenerated Problem via Minty's Lemma. Acta Mathematica Sinica, English Series, 24, 431-438. https://doi.org/10.1007/s10114-007-0970-4

[7] Akdim, Y., Azroul, E. and Benkirane, A. (2001) Existence Solutions for Quasilinear Degenerated Elliptic Equations. Electronic Journal of Differential Equations, 2001, $1-19$.

[8] Abdellaoui, B. and Peral, I. (2003) Existence and Nonexistence Results for Quasilinear Elliptic Equations Involving the $p$-Laplaces with a Critical Potential. Annali di Matematica Pura ed Applicata, 182, 247-270. https://doi.org/10.1007/s10231-002-0064-y

[9] Wen, G., Zhang, Y. and Chen, D. (2014) Approximate Solutions to the Discontinuous Riemann-Hilbert Problem of Elliptic Systems of First Order Complex Equations. Applied Mathematics, 5, 1546-1556. https://doi.org/10.4236/am.2014.510148

[10] Fan, X.L. and Zhang, Q.H. (2003) Existence for $p(x)$-Laplacien Direchlet Problem. Nonlinear Analysis, 52, 1843-1852. https://doi.org/10.1016/S0362-546X(02)00150-5

[11] Fan, X.L. and Zhao, D. (1998) On the Generalized Orlicz-Sobolev Space $W^{k, p(x)}(\Omega)$. Journal of Gansu Education College, 12, 1-6.

[12] Kovácik, O. and Rákosnik, J. (1991) On Spaces $L^{p(x)}$ and $W^{k, p(x)}$. Czechoslovak Mathematical Journal, 41, 592-618. https://doi.org/10.21136/CMJ.1991.102493

[13] Zhao, D., Qiang, W.J. and Fan, X.L. (1997) On Generalized Orlicz Spaces $L^{p(x)}(\Omega)$. Journal of Gansu Sciences, 9, 1-7.

[14] Drabek, P., Kufner, A. and Mustonen, V. (1998) Pseudo-Monotonicity and Degenerated or Singular Elliptic Operators. Bulletin of the Australian Mathematical So- 
ciety, 58, 213-221. https://doi.org/10.1017/S0004972700032184

[15] Azroul, E., Barbara, A., Elekhlifi, M. and Rhoudaf, M. (2012) T-p(x)-Solutions for Nonlinear Elliptic Equations with an $L^{1}$-Dual Datum. Applicationes Mathematicae, 39, 339-364. https://doi.org/10.4064/am39-3-8 